\title{
A LOCAL DIFFERENTIAL QUADRATURE METHOD WITH VARIABLE SHAPE MULTIQUADRICS: TESTS ON POISSON EQUATION AND FLUID DYNAMICS USING CONSISTENT CLOUD REFINEMENTS
}

\author{
J. R. da Silva ${ }^{a}$, \\ L. G. C. Santos ${ }^{b}$, \\ and N. Manzanares Filho \\ ${ }^{a}$ Universidade Federal do Acre \\ Rodovia BR 364, Km 04, Distrito Industrial \\ Rio Branco - AC, Brasi \\ jrogerio@ufac.br \\ ${ }^{\mathrm{b}}$ Universidade Federal de Itajubá \\ ABSTRACT \\ A meshless Local Differential Quadrature Method for solving partial \\ differential equations is presented in this paper. It is based in a point cloud \\ discretization and local supports. A basis set of Multiquadric functions is \\ employed for determining the weight coefficients in derivative \\ approximations. Tests with the Poisson equation are presented for verifying \\ the converge behavior of the method in Clouds with Unstructured \\ Generation (CUG's). A consistent refinement procedure for varying the \\ multiquadric shape parameter between local supports is proposed. The \\ method is finally applied for solving the classical benchmark problem of \\ natural convection in a square cavity. Satisfactory results were obtained in \\ comparison with the reference literature.
} Instituto de Engenharia Mecânica Itajubá, MG, Brasil.

Received: June 3, 2016 Revised: September 30, 2016 Accepted: October 31, 2017

Keywords: local differential quadrature method with multiquadrics, clouds with unstructured generation

\section{NOMENCLATURE}

CUG Clouds with Unstructured Generation

$c \quad$ Shape parameter of multiquadric function

c Shape factor

$D \quad$ Diameter of the minimal circle enclosing all knots in the supporting

$d \quad$ Dimension of the set $\mathbb{R}$

$f \quad$ Function obtained through the analytical solution

LDQM Local Differential Quadrature Method

$m \quad$ Order of the derivative

$N \quad$ Number of cloud points

$n_{s} \quad$ Number of points of the local support

$R a \quad$ Rayleigh number

SOR Method Sucessive Over-Relaxation

$\mathbb{R}^{d} \quad$ Set of sll ordered $d$-tuples of real numbers

$u \quad$ Analytical solution

$w \quad$ Weights

$x, y \quad$ Cartesian coordinates

Greek symbols

$\alpha \quad$ Adjustment parameter, $\alpha=\left(\underline{c}^{2} n_{s}{ }^{2} n_{s}\right) / N$

$\Delta \quad$ Differential operator

$\varepsilon \quad$ Relative error

$\begin{array}{ll}\theta & \text { Temperature } \\ \varphi & \text { Multiquadric function } \\ \psi & \text { Stream function } \\ \Omega & \text { Square domain } \\ \omega & \text { Vorticity }\end{array}$

\section{Subscripts}

$i \quad$ Global index

$j, k \quad$ Local indexes

\section{INTRODUCTION}

The meshless methods have gained substantial attention among the numerical methods in recent years. The advantage of not being restricted to a preexisting connection between dots in the computational domain, make these very attractive both in the area of applied sciences and in engineering methods. Among these methods, the method of Differential Quadrature Local has become quite prominent among without tools-existing mesh in computational fluid dynamics. This method was based on the ideas of Classical quadrature However, this method applies only to get derived from any order. The method was developed by Bellman et al. (1972) and had great contribution Kansa (1990) to innovatively make use of Radial Basis Function 
(RBF) in global approaches. The insertion of these functions by Differential Quadrature method allowed both flexibility regarding the use or not knitting, the univariate method as characteristic. Moreover, according to da Silva (2014) the multiquadric FBR possesses one shape parameter whose value can be chosen optimally based on the number of points of local support. Several papers have been published with the MQDL-FBR applications to computational

fluid dynamics, particularly in studies of bidimensional incompressible flow.

This paper proposes tests MQDL-FBR in numerical solution of the Poisson equation in two dimensions, focusing on a consistent technique to the refinement of the approach using cloud parameter variable as proposed by Shu et al. (2003), as well as the application to a classic problem of heat transfer by natural convection in a square cavity.

\section{LOCAL DIFFERENTIAL QUADRATURE METHOD WITH RADIAL BASIS FUNCTIONS}

The Local Differential Quadrature Method (LDQM) was introduced by Shu et al. (2003) for solving differential equations in a meshless approach. It consists in approximating the spatial derivatives of a certain function at a point $\mathrm{x}$ in $\mathbb{R}^{d}$ by means of a weighted sum of function values in $n_{s}$ points close to $x$, including $x$ itself (the support of $x$ ). Considering in Fig. 1 a cloud of $N$ discrete points the $m$ order derivative of a smooth function $f: \mathbb{R}^{d} \rightarrow \mathbb{R}$ in a point $x^{i}$ (reference point of $i$ support, $i=1, \ldots, N$ ) is then written:

$$
\frac{\partial^{m} f\left(x_{i}\right)}{\partial x^{m}}=\sum_{j=1}^{n_{s}} w_{i, j}^{m x} f\left(x_{j}\right), i=1,2, \ldots, N
$$

Where $w_{i, j}^{m x}$ are the respective weights whose previous determination is a crucial step of the method. In Eq. (1), the index $i$ indicates a reference point of the cloud (global numeration: $i=1, \ldots, N$ ) while $j$ is a index for the corresponding supporting points (local numeration: $j=1, \ldots, N$ ).

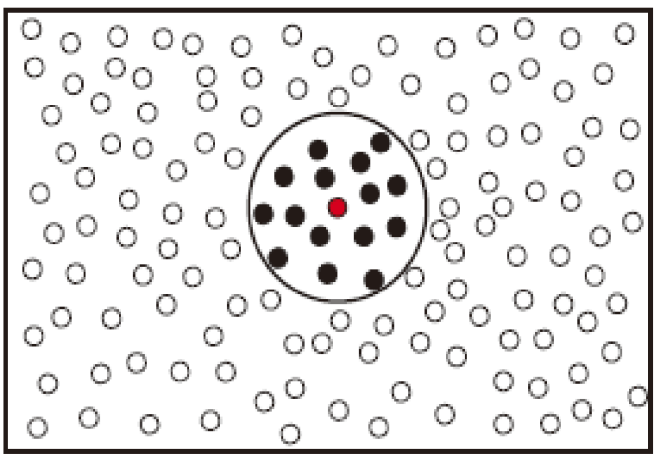

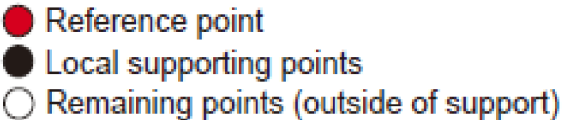

Figure 1. Point cloud highlighting the local supporting points (black), the respective reference point (red) and the remain- ing points (outside of support).

The determination of weights was made in this work by the procedure proposed by Ding et al. (2005). A basis set of $n_{s}$ Multiquadric functions $\varphi k$ centered at the supporting points is used for testing Eq. (1), $\varphi k(x)=\sqrt{\left(\left\|x-x_{k}\right\|_{2}\right)^{2}+c^{2}}, k=1 \ldots, n_{s}$, where $c$ is a shape parameter and $\|$.$\| represents the$ Euclidian norm. The following $n_{s} \times n_{s}$ system of linear algebraic equations for the unknown weights results:

$$
\begin{array}{r}
\frac{\partial^{m} \varphi k\left(x_{i}\right)}{\partial x^{m}}=\sum_{j=1}^{n_{s}} w_{i, j}^{m x} \varphi k\left(x_{j}\right), k=1,2 \ldots, n_{s} \\
\text { for } i=1, \ldots N
\end{array}
$$

Various criteria for defining the support of a certain reference point can be conceived. The simple and effective criterion of using the $n_{s}$ points closer of the reference point including itself was adopted in this work. The same $n_{s}$ was used for all supports.

There will be $N$ inear systems to be solved for the whole cloud, i.e., one system for each reference point and their respective supporting points. After solving these local systems for obtaining the weights in all supports and for all derivatives of interest, it becomes possible to discretize a partial differential equation in terms of the unknown function values at the $N$ points of the cloud. After applying boundary and initial conditions and discretizing temporal derivatives (if necessary), the global system can be solved by conventional methods. For the Poisson equation addressed in the next section, the method of Successive Over-Relaxation (SOR) was chosen.

\section{LDQM TESTS WITH POISSON EQUATION}

Some numerical experiments were performed for analyzing the convergence and accuracy of LDQM. The 2D Poisson equation in a square domain was considered, $\Omega=\{(x, y) \in \mathbb{R} 2: 0 \leq x \leq 1 \mathrm{e}$ $0 \leq y \leq 1\}$ :

$$
\nabla^{2} u=f(x, y)
$$

The Dirichlet boundary condition was applied, i.e., $u=u_{\text {exact }}$ at the boundary points. An exact 
(analytical solution) $u=u_{\text {exact }}(x, y)$ is chosen as a reference and the corresponding r.h.s. function $f(x, y)$ follows from Eq. (3) and is used for obtaining the numerical solutions. The exact solution used by Shu et al. (2003) was considered in this work:

$$
u_{\text {exact }}(x, y)=\frac{\frac{5}{4}+\cos (5.4 y)}{6=6(3 x-1)^{2}}
$$

The relative error used for comparison was defined by:

$$
\|\varepsilon\|=\frac{\sqrt{\sum_{i=1}^{N_{\text {int }}}\left(u_{\text {num }}-u_{\text {exact }}\right)_{i}^{2}}}{\sqrt{\sum_{i=1}^{N_{\text {int }}}\left(u_{\text {exact }}\right)_{i}^{2}}}
$$

Clouds with Unstructured Generation (CUG's) were employed for the tests. They were extracted from finite element meshes generated by means of Matlab PDETool. An important aspect was to dispense the use of points outside the domain (ghost points) - differently of Santos (2012). Structured meshes close to the boundary, as those employed by Shu et al. (2003), were also not used. Figure 2 illustrate one of the CGU's used in the tests.

The procedure proposed by Shu et al. (2003) for varying the shape factor between the supports was initially tested. This procedure aims to mitigate the relative size effects of the supports by setting a nondimensional shape factor $\bar{c}$ and then varying the shape factor $c$ proportionally to the support size:

$$
c_{i}=\bar{c} D_{i}
$$

where $D_{i}$ is the diameter of the minimum circle that embraces all the points of the $i$ support.

The first tests focused in evaluating the effect of the cloud refinement for fixed values of the nondimensional shape factor. CUG's with numbers of points $N$ equal to 595, 2060, 6050 and 10435 were tested for values of $\bar{c}^{2}$ equal to $1.5,2.0,2.5$, 3.0, and 3.5. Supports with $n_{s}$ equal to 9 and 15 were addressed.

The results are shown in Fig. 3. One observes that this cloud refinement procedure is not consistent in general. Increasing the number of points of the cloud do not produced a decrease in the relative error. On the contrary, the error clearly increases with the refinement for $n_{s}=9$. The explanation for this inconsistency is simple: it is known that the decrease of (dimensional) shape factor $\mathrm{c}$ tends to worsen the results (Ding et al. (2005)). And this is what happens when the cloud is refined with $\bar{c}^{2}$ fixed: support sizes decrease and respective $c$ values also decrease, according to Eq. (6). Thus the trend of decreasing the error as the cloud is refined may be annulated or even inverted by the decrease of $c$.

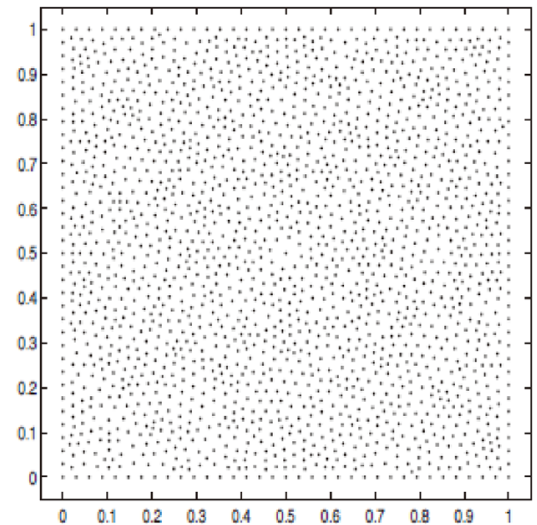

Figure 2. Cloud with Unstructured Generation (CUG) of 2060 points.
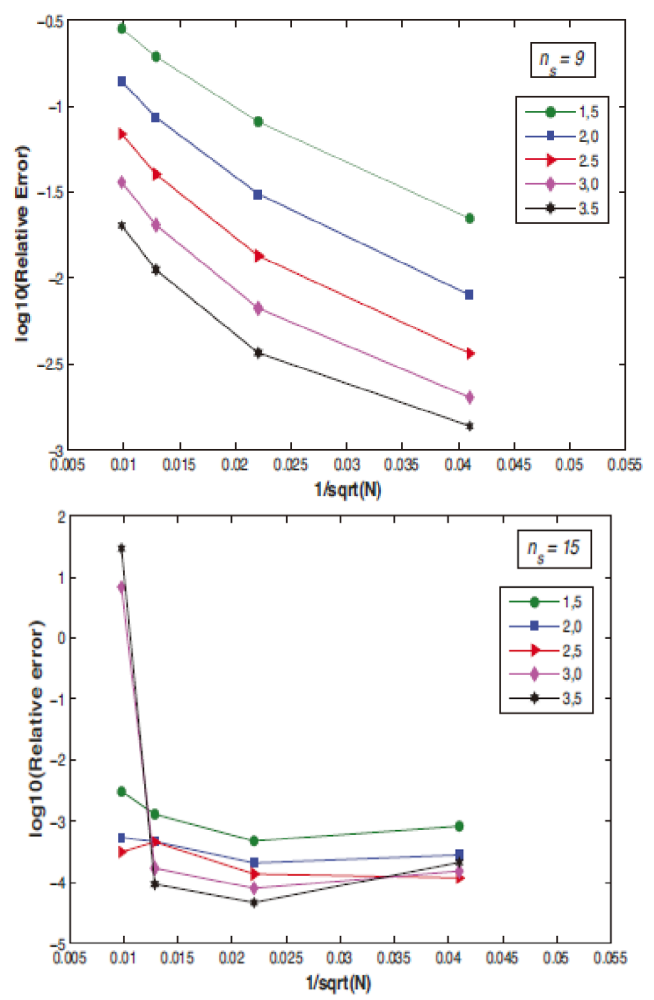

Figure 3. Effect of the cloud refinement ( $N$ variation) on the relative error for various values of $\bar{c}^{2}$ and supports with $n_{s}=9$ and $n_{s}=15$.

In order to make the cloud refinement consistent it appears necessary to vary $\bar{c}$ in such manner that the distribution of $c$ do not vary substantially as the number of points of the cloud is varied. Thus, by Eq. (6), $c$ must roughly vary with $1 / D_{i}$. On the other 
hand, for a certain domain, the support area $\left(\sim D_{i}^{2}\right)$ is proportional to ns and inversely proportional to $N$. It follows that:

$$
\begin{aligned}
c_{i}=\bar{c} D_{i} \text { where } \bar{c}^{2}=\alpha\left(\frac{N}{n_{s}}\right) \text { with } \alpha & =\left(\frac{\bar{c}^{2} n_{s}}{N}\right) \\
\text { and } i & =1,2, \ldots, N
\end{aligned}
$$

where $\alpha$ is an adjustment parameter that can be evaluated by using reference values of $\bar{c}, n_{s}$, and $N$.

The numerical tests were performed again but now with fixed values of $\alpha$. Such values were calculated by Eq. (7) with reference values of $\left(n_{s}\right)_{\text {ref }}=15$ and $(N)_{\text {ref }}=10435$ and reference values of $\left(\bar{c}^{2}\right)_{\text {ref }}$ equal to $1.5,2.0,2.5,3.0$, and 3.5.

The results are shown in Fig. 4. One observes now a consistent decrease of the relative error with the cloud refinement.

\section{APPLICATION OF THE LDQM TO PROBLEM OF FLUID DYMAMICS}

The LDQM was applied for solving the classical benchmark problem of natural convection in a square cavity with horizontal adiabatic walls and isothermal vertical walls subject to a thermal gradient. The stream function $(\psi)$ - vorticity $(\omega)$ formulation was used in nondimensional form (temperature $\theta$ ). The equations were omitted.
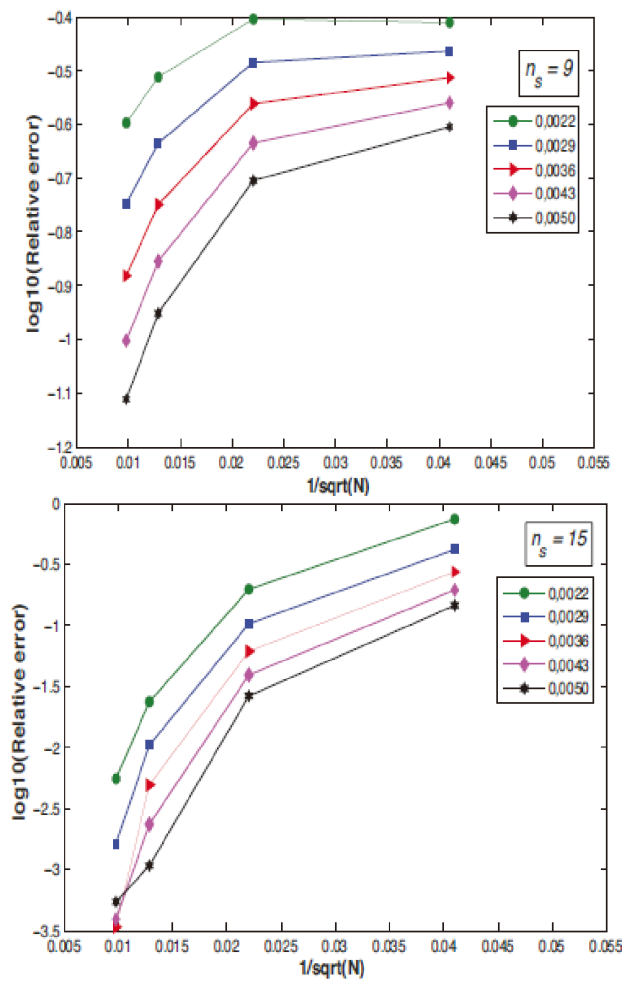

Figure 4. Effect of the cloud refinement ( $N$ variation) on the relative error for various values of $\alpha$ and supports with $n_{s}=9$ and $n_{s}=15$.

Values of Rayleigh number $R a$ equal to $10^{4}, 10^{5}$ and $10^{6}$ were tested using CUG's with 5421 and 10020 points and local supports with 9 and 15 points. The adjustment parameter $\alpha$ was fixed as 0.004 . The vorticitiy and energy equations were advanced in time by means of the explicit Euler scheme. In each time step, the Poisson equation was solved by the SOR method.

Results for the average Nusselt number at the isothermal hot wall are presented in Table 1 in comparison with reference results of Davis (1983) and Shu et al. (2003). One observes that substantially better results were obtained with the support of 15 points than with the support of 9 points.

Table1. Average Nusselt number.

\begin{tabular}{c|ccc|ccc}
\hline & \multicolumn{3}{|c|}{$\mathrm{n}_{\mathrm{s}}=9$} & \multicolumn{3}{c}{$\mathrm{n}_{\mathrm{s}}=15$} \\
Nuvens & $\mathrm{Ra}=10^{4}$ & $\mathrm{Ra}=10^{5}$ & $\mathrm{Ra}=10^{6}$ & $\mathrm{Ra}=10^{4}$ & $\mathrm{Ra}=10^{5}$ & $\mathrm{Ra}=10^{6}$ \\
\hline 5421 & 2.385 & 4.084 & 8.182 & 2.287 & 4.587 & 8.796 \\
10020 & 2.285 & 4.232 & 8.266 & 2.249 & 4.562 & 8.791 \\
$\begin{array}{c}\text { Davis(1983) } \\
\text { Shu et al. } \\
\text { (2003) }\end{array}$ & 2.240 & 4.573 & 8.932 & 2.240 & 4.573 & 8.932 \\
\hline
\end{tabular}

\section{CONCLUSIONS}

The present article highlighted the study of a technique consistent with the use of the shape parameter variable of the LDQM as well as, the application of this method in a two-dimensional problem of heat transfer by natural convection.

The technique of Shu et al. (2003), whose values of the form parameter $(c)$ vary with the size of the local support, however, since the cloud of points is refined, the values of $D$ are reduced and consequently those of $c$, the accuracy of the MQDL. To correct this inconsistency, we proposed the adjustment parameter $\alpha$, whose values depend on $\underline{c}$, $n_{s}$ and $N$. This proposed technique was validated through the results obtained in numerical simulation for natural convection in the square cavity. And, although no point structure was used at the border of the computational domain, the results were satisfactory and agree well with those obtained in the based on mesh by other researchers.

Differently from previous approaches to LDQM, the potential of LDQM in the problems of a complex nature, especially without mesh. However, since other entities of the method as ns value and the shape of the support influence its accuracy, it is still necessary to development of LDQM, whose research still remains incipient.

\section{ACKNOWLEDGEMENTS}

The authors thanks CAPES and FAPEMIG for financial support. 


\section{REFERENCES}

Davis, G., 1983, Natural Convection of Air in a Square Cavity: a Benchmark Numerical Solution, International Journal for Numerical Methods in Fluids, Vol. 3, pp. 249-263.

Ding, H., Shu, C., and Tang, D., 2005, Error Estimates of Local Multiquadric-Based Differential Quadrature (LMQDQ) Method Through Numerical Experiments, International Journal for Numerical Methods in Engineering, Vol. 63, pp. 1513-1529.

Santos, L. G. C., 2012, Método da Quadratura Diferencial Local com Funções de Base Radial Aplicados em Problemas de Dinâmica dos Fluidos e Transferência de Calor, Doctoral Thesis, Universidade Federal de Itajubá, Itajubá, MG, Brasil. (in Portuguese)

Shu, C., Ding, H., and Yeo, S., 2003, Local Radial Basis Function-Based Differential Quadrature Method and its Application to Solve TwoDimensional Incompressible Navier-Stokes Equations, Computer Methods in Applied Mechanics and Engineering, Vol. 192, pp. 941-954. 